\title{
THE EFFECT OF ANION SUBSTITUTION IN NICKEL HEXAMMINE SALTS
}

\author{
H. BLANK and G.J. KEARLEY \\ Institut Laue-Langevin, 156X, F-38042 Grenoble cedex, France
}

\begin{abstract}
Several metal hexammine salts show rotational tunnelling of the $\mathrm{NH}_{3}$ groups in their low-temperature phases /1,2/. This $\mathrm{NH}_{3}$ tunnelling on one hexammine cation is strongly influenced by $\mathrm{NH}_{3}$ groups of the neighbouring cations. It is important to elucidate whether the anions play a direct rôle in determining the rotational barrier or whether changes in the barrier result simply from changes in the lattice parameter. To this end we have started a study of $\mathrm{Ni}\left(\mathrm{NH}_{3}\right)_{6} \mathrm{l}_{2}$ in which we replaced varying amounts of the iodide ions with bromides as substitutional impurities. We synthesised mixtures of $\mathrm{Ni}\left(\mathrm{NH}_{3}\right)_{6}(\mathrm{I}, \mathrm{Br})_{2}$ with $1,5,10,20$ and 50 atom\% $\mathrm{Br}$. A normal distribution of next neighbours fits the low-resolution neutron scattering data well. The presence of the bromide ions as substitutional impurities creates regions of "negative pressure" in the nickel hexammine iodide lattice.
\end{abstract}

\section{Introduction}

Several metal hexammine salts, such as: nickel hexammine iodide and nickel hexammine bromide, show rotational tunnelling of the $\mathrm{NH}_{3}$ groups in their low-temperature phases. Three peaks due to rotational tunnelling-transitions of the $\mathrm{NH}_{3}$ ligands in $\mathrm{Ni}\left(\mathrm{NH}_{3}\right)_{6} \mathrm{l}_{2}$ can be observed at 80,68 and $45 \mu \mathrm{eV} / 1 /$, whilst the analogous bromide salt has a single broad peak at $8 \mu \mathrm{eV} / 2$. The decrease of the ionic radii of the anion seems to play an important rôle in increasing the barrier height. To investigate this point further we have started a study of $\mathrm{Ni}\left(\mathrm{NH}_{3}\right)_{6} \mathrm{I}_{2}$ in which we substituted varying amounts of iodide ions with bromides. It appears that it is possible to prepare mixed crystals of $\mathrm{Ni}\left(\mathrm{NH}_{3}\right)_{6}\left(\mathrm{I}_{2-2 x}, \mathrm{Br} 2 \mathrm{x}\right)$ over the entire concentration range. The $X$-ray powder diagrams show that this is certainly true between the pure iodide salt and up to $50 \%$ bromide-ion substitution.

\section{Experimental}

Samples were prepared by adding a solution of the appropriate mixture of $\mathrm{NH}_{4} \mathrm{Br}$ and $\mathrm{NH}_{4}$ l to $\mathrm{Ni}\left(\mathrm{NH}_{3}\right)_{6}(\mathrm{OH})_{2}$ solution and filtering off the precipitated mixed crystals. Powder X-ray diffraction patterns were collected to check for crystallinity and unit-cell parameters of each of 
the samples. Inelastic neutron scattering (INS) data were collected on the time-of-flight instrument IN5 at the Institut Laue-Langevin in France $3 /$. The incident wavelengths used were 6 and $13 \AA$, giving an energy resolution of 63 and $6 \mu \mathrm{eV}$, respectively. All spectra were measured at $1.8 \mathrm{~K}$ using a standard pumped liquid-helium cryostat. The spectra at different scattering angles were summed to a single spectrum. Data treatment was carried out using standard algorithms.

\section{Results and Discussion}

Fig. 1 shows the INS spectra of the mixed $\mathrm{Ni}\left(\mathrm{NH}_{3}\right)_{6}\left(\mathrm{I}_{2-2 x} \mathrm{Br} 2 \mathrm{x}\right)$ at concentrations of $x=0.01,0.05,0.1,0.2,0.35,0.5$ measured at an average $Q$ of $1.7 \AA^{-1}$. The shoulder at 0.6 $\mathrm{meV}$, which can be observed at low concentrations, gradually disappears with increasing $\mathrm{Br}$ concentration. Similar effects were found in $\mathrm{K}_{1-\mathrm{x}}\left(\mathrm{NH}_{4}\right)_{\mathrm{x}} \mathrm{I} / 4 /$ and methane in krypton $/ 5 /$. The $x=0.5$ spectrum resembles a "quasielastic" spectrum. The differing intensities of the shoulders on either side of the elastic peak are due to the detailed balance.

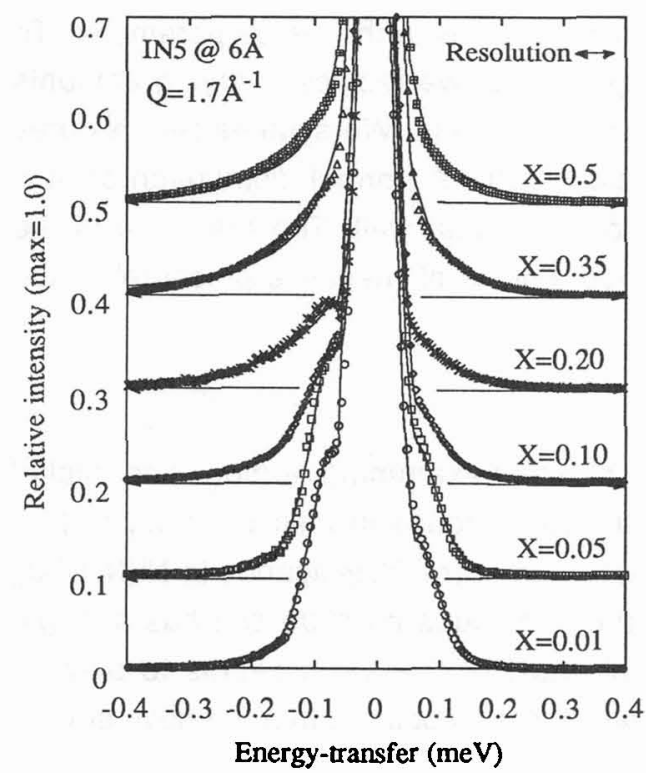

Figure 1: Inelastic neutron scattering spectra of mixed crystals of $\mathrm{Ni}\left(\mathrm{NH}_{3}\right)_{6}\left(I_{2-2 x} \mathrm{Br}_{2 x}\right)$. Data were collected at a sample temperature of $1.8 \mathrm{~K}$ with an energy resolution of $63 \mu \mathrm{eV}$.

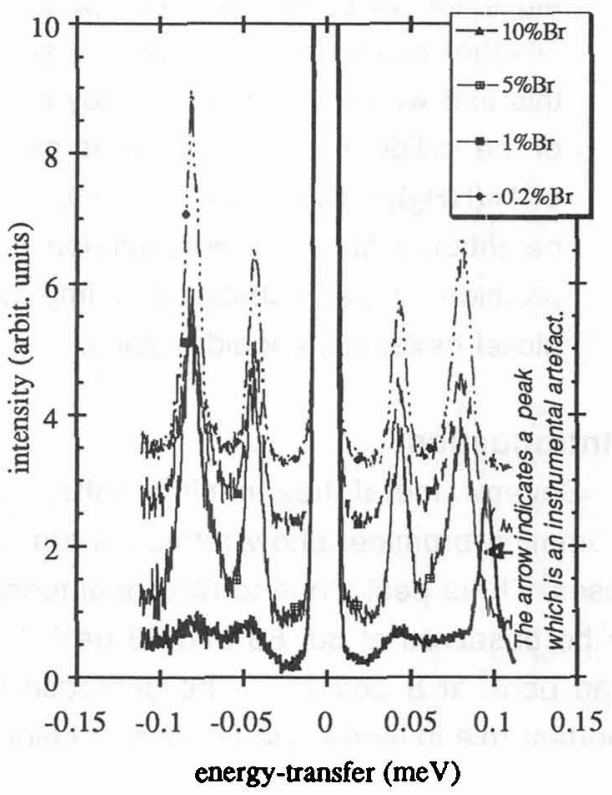

Figure 2: High resolution data of

$\mathrm{Ni}\left(\mathrm{NH}_{3}\right)_{6}\left(\mathrm{I}_{2-2 x}, \mathrm{Br}_{2 x}\right)$ with $\mathrm{x}=0.002,0.01$,

0.05 , and 0.1 . The sample temperature was $1.8 \mathrm{~K}$ and the energy resolution $6 \mu \mathrm{V}$.

The high resolution data, shown in fig. 2, only cover a small spectral range (energy resolution $6 \mu \mathrm{eV}, \mathrm{Q}$ of $0.25 \AA^{-1}$ ). As the fraction of $\mathrm{Br}$ increases the peaks in the spectrum decrease and are almost vanished at 10 atom\%.

In $\mathrm{Co}\left(\mathrm{NH}_{3}\right)_{6}\left(\mathrm{PF}_{6}\right)_{2}$ the $\mathrm{NH}_{3}$ groups are nearly free rotating indicating that intra-ionic $\mathrm{NH}_{3}$ interactions are very small $/ 6 \%$. In the nickel hexammine iodide and bromide the barrier height 
is much higher from which it follows that the rôle of the anion is in some way dominant. Each cation is surrounded by 8 anions at approximately the same distance. The distribution of the $\mathrm{Br}$ - in the closest coordination at different sample compositions will follow the coefficients of a binomial distribution. For a given concentration of $\mathrm{Br}$ the number of nearest neighbours for a $\mathrm{Ni}\left(\mathrm{NH}_{3}\right)_{6}{ }^{2+}$ ion is shown in fig. 3 (note that at an atom-fraction of $0.5 \mathrm{Br}$ there is a symmetry for this binomial distribution).

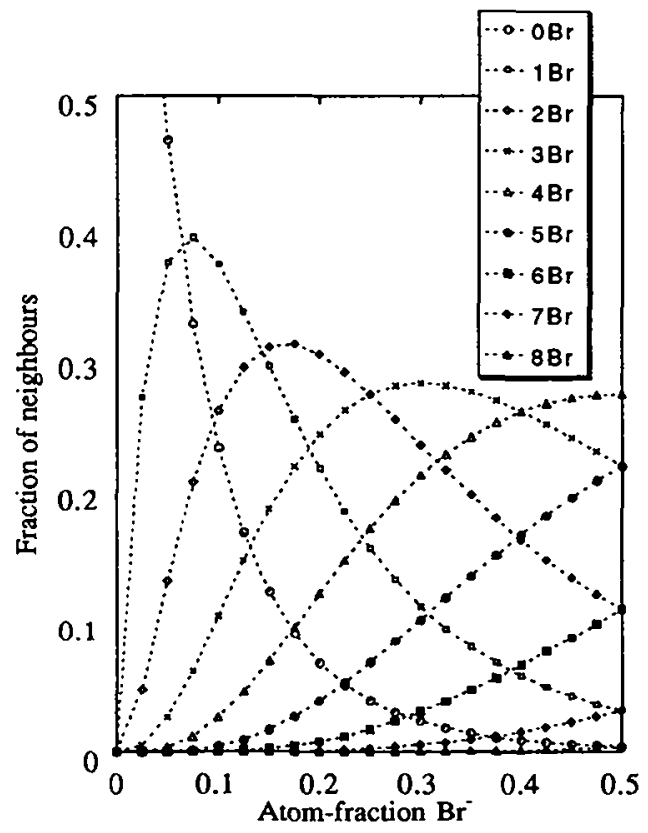

Figure 3: Relative concentrations of eight nearest neighbours between 0 and 0.5 atom fraction.

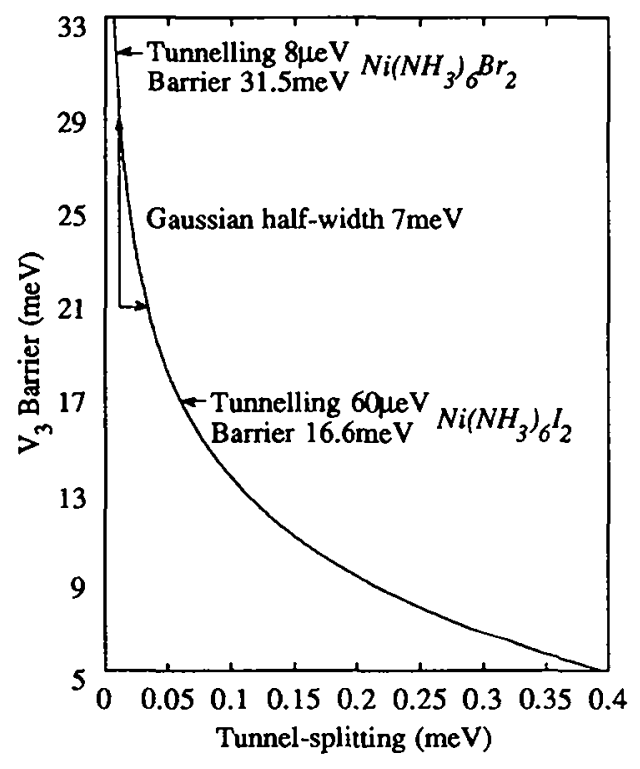

Figure 4: Tunnel splitting versus the barrier height of a threefold rotational potential. The projection of the Gaussian distribution of barrier heights onto the "tunnelling-axis" is shown.

The true number of different anion surroundings of the cation is much greater than eight and each of these will present its own hindrance potential to the $\mathrm{NH}_{3}$ rotations on the hexammine ion. As a first approximation we have treated these distributions as being Gaussian. Therefore, it can be expected that at each concentration the observed spectrum will be described by a Gaussian distribution of barrier heights with the widths and positions to be determined from the experiment. It is important to note that a Gaussian distribution of barrier heights does not lead to Gaussian shaped peaks in the energy spectrum. Fig. 4 shows that the tunnel splitting varies almost exponentially with the barrier height. The model used was a Gaussian distribution of barrier heights transformed to the energy scale by diagonalisation of the Hamiltonian given below:

$$
\mathcal{H}=\mathrm{B}\left[\left(\delta^{2} / \delta \theta^{2}\right)\right]+V(\theta)
$$

where $B$ is the rotational constant, and $V(\theta)$ the periodic potential. The resulting energy spectrum was convoluted with the measured instrumental resolution to produce the final 
calculated spectrum. Only three parameters were fitted: the Gaussian position, width and an overall scale factor. The solid line in fig. 1 shows that the resulting fit to the data is rather good.

The energy eigenvalues of the Hamiltonian are plotted against the potential barrier height in fig. 4. The points corresponding to pure $\mathrm{Ni}\left(\mathrm{NH}_{3}\right)_{6} \mathrm{I}_{2}$ with a barrier height of $16.6 \mathrm{meV}$ and the $\mathrm{Ni}\left(\mathrm{NH}_{3}\right)_{6} \mathrm{Br}_{2}$ with a barrier height of $8 \mathrm{meV}$ are indicated by arrows. The trend in moving from the pure iodide salt to the pure bromide is a progressive increase in barrier height. There is also a progressive increase in the width of the Gaussian distribution of barrier heights up to a maximum value of at around $50 \%$. Although we have no data between 50 and 99 atom\% $\mathrm{Br}$, we presume that there would be a progressive decrease in width over this range. Fig. 5 shows the variation of the mean barrier height of a threefold rotational potential with increasing bromide-ion concentration in $\mathrm{Ni}\left(\mathrm{NH}_{3}\right)_{6} \mathrm{I}_{2}$. These data points lie approximately on a straight line, although deviations from this straight line are to be expected, since the occupation of the different anion environments will vary rapidly at around 0 and 100 atom\% $\mathrm{Br}$ with a flatter region around $50 \%$. This can be deduced from fig. 3 in that the steepest parts of the concentration curves are closest to the pure compounds.

Our X-ray data show the lattice

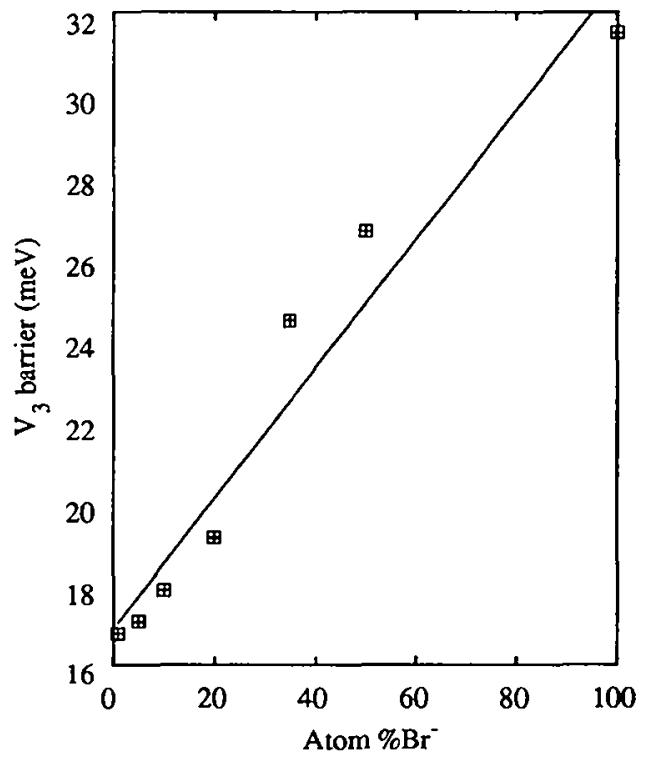

Figure 5: Variation of the threefold potential $V_{3}$ with atom\% $\mathrm{Br}^{-}$in $\mathrm{Ni}\left(\mathrm{NH}_{3}\right)_{6} I_{2}$. parameter of pure nickel hexammine bromide to be $4 \%$ smaller than that of pure nickel hexammine iodide. Only a $0.7 \%$ decrease is found in going from the pure iodide salt to 50 atom\% bromide ion which leaves the majority of the lattice shrinkage to occur in passing from $\mathbf{5 0}$ atom\% bromide ion to the pure bromide salt. Unfortunately we have no data in this concentration range at present.

The near linearity of the change in rotational barrier between $0.01 \%$ and $50 \%$ atom\% $\mathrm{Br}$ - shown in fig. 5 is somewhat surprising in view of the almost negligible change in the lattice parameter. Were the barrier given by simple $\mathrm{H}$-atom/ $\mathrm{H}$-atom potentials between neighbouring hexammine ions we would expect very little change in the mean barrier height between $0 \%$ and $50 \% \mathrm{Br}$, since the lattice parameter scarcely changes. That this barrier increases almost linearly leads to the conclusion that the anion plays an important direct rôle in determining the rotational potential of the $\mathrm{NH}_{3}$ groups which is independent, at least to some degree, of the distance between cations. 
In the near pure salts individual tunnelling peaks are resolved, see fig. 2 and there is clearly an error associated with treating these multiplets as a smooth distribution. However, for most of the mixtures the components of the multiplet are so broad that this approximation works well as shown in fig. 6. At present, we have no quantitative model for the increase in distribution-width of the rotational barrier between $0 \%$ and 50 atom $\% \mathrm{Br}^{-}$. It is particularly interesting that as the width of the distribution increases a small fraction of the $\mathrm{NH}_{3}$ rotors experience a rotational barrier which is very much lower than that of the pure iodide salt. For example, the 35 atom\% $\mathrm{Br}$ sample still shows measurable intensity above background at $400 \mu \mathrm{eV}$, which corresponds to a threefold barrier of about $5 \mathrm{meV}$. This is less than a third of the

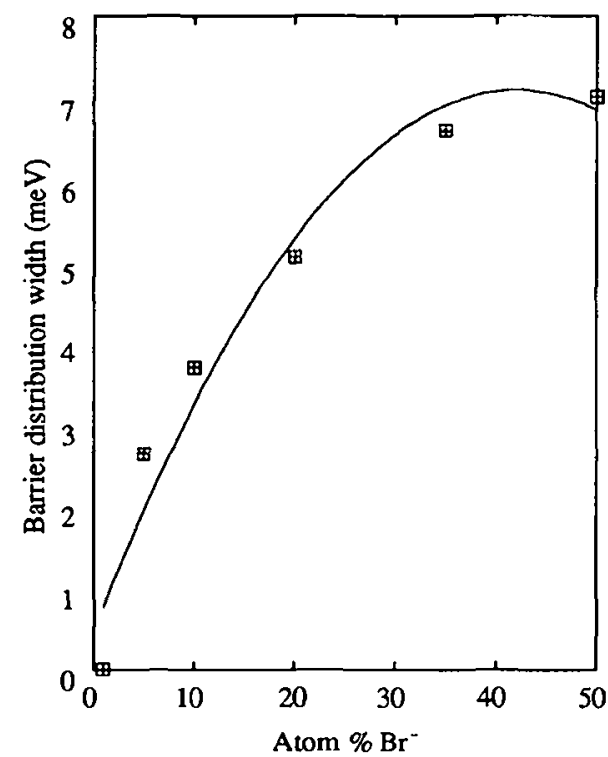

Figure 6: Variation of the width of the distribution of rotational barrier heights with change of $\mathrm{Br}^{\circ}$ concentration in $\mathrm{Ni}\left(\mathrm{NH}_{3}\right)_{6} l_{2}$. barrier height of the pure iodide salt and represents a near free rotor. Possibly this reflects regions in the disordered crystal of reduced "pressure".

\section{Acknowledgement}

We are grateful to Dr. C. Cohen Addad for making the X-ray measurements possible at the Grenoble university.

\section{References}

11/ H. Blank and G. J. Kearley, J. Chem. Phys. 87 (1987) 6809

12/ W. Press and M. Prager, J. Chem. Phys. 67 (1977) 5752

13/ H. Blank and B. Maier. The Yellow Book: Guide to neutron research facilities at the $\mathrm{LLL}$, Grenoble (1988)

14/ C. Bostoen, G. Coddens, and W. Wegener, J. Chem. Phys. 91 (1989) 6337

15/ B. Asmussen, Symp. on Quantum Aspects of Molecular Motions in Solids, 3-6 Apr 91, Rottach-Egern

/6/ H. Blank and G.J. Kearley, Physica B, in press 